\title{
First operation of a double phase LAr Large Electron Multiplier Time Projection Chamber with a two-dimensional projective readout anode
}

\author{
A. Badertscher, A. Curioni, L. Knecht, D. Lussi, A. Marchionni, \\ G. Natterer, F. Resnati, A. Rubbia, T. Viant \\ Institute for Particle Physics, ETH Zurich, 8093 Zurich, Switzerland
}

\begin{abstract}
We have previously reported on the construction and successful operation of the novel double phase Liquid Argon Large Electron Multiplier Time Projection Chamber (LAr LEM-TPC). This detector concept provides a 3Dtracking and calorimetric device capable of adjustable charge amplification, a promising readout technology for next-generation neutrino detectors and direct Dark Matter searches.

In this paper, we report on the first operation of a LAr LEM-TPC prototype - with an active area of $10 \times 10 \mathrm{~cm}^{2}$ and $21 \mathrm{~cm}$ drift length - equipped with a single $1 \mathrm{~mm}$ thick LEM amplifying stage and a two dimensional projective readout anode. Cosmic muon events were collected, fully reconstructed and used to characterize the performance of the chamber. The obtained signals provide images of very high quality and the energy loss distributions of minimum ionizing tracks give a direct estimate of the amplification. We find that a stable gain of 27 can be achieved with this detector configuration corresponding to a signal-over-noise ratio larger than 200 for minimum ionizing tracks. The decoupling of the amplification stage and the use of the 2D readout anode offer several advantages which are described in the text.
\end{abstract}

Key words:

Liquid argon, Pure argon, Double phase, LAr TPC, TPC, LEM, THGEM, GEM, Calorimetry, Tracking, Gaseous Detector 


\section{Introduction}

The Liquid Argon Large Electron Multiplier Time Projection Chamber (LAr LEM-TPC) is a novel kind of double phase (liquid-vapor) noble gas TPC with adjustable gain [1]. The chamber is a precise tracking device that allows to reconstruct three-dimensionally the position and the morphology of ionizing tracks, $d E / d x$ information with high sampling rate, and it acts as high resolution calorimeter for contained events. Thanks to the gain, each volumetric pixel (voxel) is reconstructed with high signal-to-noise ratio and consequently a low energy deposition threshold is possible. In addition charge amplification reduces the impact of charge dilution due to the longitudinal diffusion of the electron cloud along the drift paths, and can be used to compensate for potential charge losses due to electronegative impurities diluted in the liquid argon. We believe that this technology is very promising for the realization of next generation underground detectors for neutrino physics and proton decay searches [2, 3, 4] and for direct Dark Matter detection [5] with imaging technique, in all cases with a significant improvement of the imaging quality compared to the single phase liquid argon TPC [6].

In this paper, we report on the first operation of a LAr LEM-TPC prototype with an active area of $10 \times 10 \mathrm{~cm}^{2}$ and $21 \mathrm{~cm}$ drift length, equipped with a single $1 \mathrm{~mm}$ thick LEM amplifying stage and a two dimensional projective readout anode.

The charge produced by ionizing particles in the liquid is drifted towards the liquid-vapor interface, where electrons are extracted to the vapor phase by means of an appropriate electric field produced by two grids. In the vapor phase, Townsend avalanche takes place in the high electric field regions confined in the LEM holes, similar to the situation of the Gas Electron Multiplier (GEM) [7]. The LEM is a macroscopic hole electron multiplier built with standard PCB techniques. The amplified charge is collected by a set of segmented electrodes, on which signals are induced.

In order to obtain a complete 3D-spatial reconstruction of ionizing events, moving charges have to induce signals on (at least) two complementary X$\mathrm{Y}$ sets of electrode strips. The $\mathrm{Z}$ (drift) coordinate is extracted from the drift time determined from the $T_{0}$ given by immersed DUV-sensitive photo detectors recording the prompt LAr scintillation light. In our previous setups [6, 1, 8], we implemented two perpendicular X\&Y views by a segmentation of the upper electrode of the LEM (X-view) and of the anode (Y-view) with $6 \mathrm{~mm}$ pitch. However, long-term operation in stable conditions with 
gain indicated that a fine segmentation of the LEM creates electric field distortions which may trigger discharges. We concluded on the necessity to decouple the charge multiplication and readout stages [8].

This separation has been achieved thanks to the use of a projective 2D charge X-Y-readout, providing two independent views with $3 \mathrm{~mm}$ effective pitch. Based on the GEM 2D readout concept [9], it consists of two perpendicular sets of strips with a $50 \mu \mathrm{m}$ thick Kapton spacer in-between. The drifting charge is collected on both sets of strips, based on the principle of charge sharing.

This new layout has several advantages: (1) the functions of amplification and of readout are decoupled, i.e. avoiding the need for a segmented LEM plane, which is more prone to electric discharges; (2) as a consequence, there is no need to install HV decoupling capacitors on the LEM electrodes; (3) the pitch of the readout is not constrained by the LEM hole size and pitch; (4) the amplified charge is effectively collected on a single plane where two perpendicular sets of strips of a two dimensional projective anode are located, simplifying the overall design; (5) with a proper strip geometry, the charge can be equally shared and collected on both readout views, therefore the signal shapes are unipolar and identical on both views, easing the reconstruction and feature extraction of the signal waveforms.

In section 2 we briefly describe the experimental setup. The chosen electric configuration is motivated in section 3. LEM and projective anode are then detailed in the sections 4 and 5 . Section 6 describes the operation of the chamber, while cosmic muon reconstruction is presented in section 7. Results on the characterization of the detector are given in section 8 .

\section{The experimental setup}

The purpose of this section is to briefly describe the 3L prototype chamber used as a test-bench setup (see Figure 1). A more detailed description can be found elsewhere [1]. Figure 2 shows a picture of the detector (left), with the corresponding schematics (right). The LAr drift volume has a cross-section of 10x10 $\mathrm{cm}^{2}$ and the distance between cathode and LAr surface equals $21 \mathrm{~cm}$. Below the cathode grid, optically transparent with a wire pitch of $5 \mathrm{~mm}$, a Hamamatsu R6237-01 photomultiplier tube, coated with the wavelength shifter tetraphenylbutadiene (TPB) [10] is positioned below a grounded protection grid. It allows to detect the liquid argon scintillation light, peaked around $128 \mathrm{~nm}$ [11]. The uniform drift field is defined by equally-spaced 


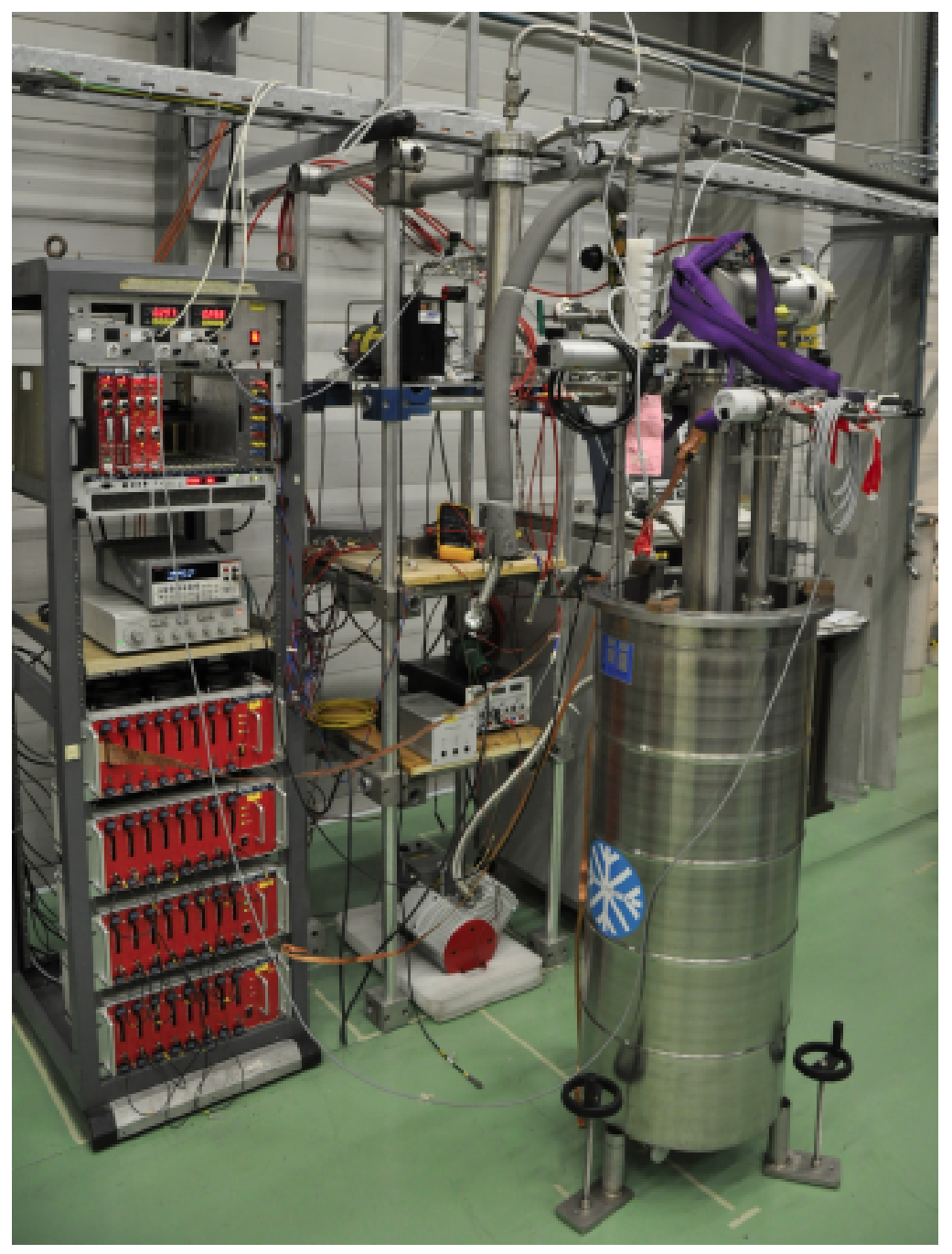

Figure 1: Overview of the 3L setup. 
rectangular field shaping rings, connected to a chain of $940 \mathrm{M} \Omega$ resistors. On top of the drift volume there are two parallel extraction grids with a wire pitch of $5 \mathrm{~mm}$ and a gap of $10 \mathrm{~mm}$. These two grids allow to apply an electric field across the LAr surface which is independent of the chosen drift field. The leveling of the LAr is done with millimeter precision by a capacity measurement of the two grids. The LEM and the $2 \mathrm{D}$ anode are mounted $1 \mathrm{~cm}$ above the upper grid and $2 \mathrm{~mm}$ apart from each other. It is worth mentioning that the $2 \mathrm{D}$ anode can be operated at high voltage. This is done (see Figure 3) by connecting each strip with a $500 \mathrm{M} \Omega$ resistor to the guard ring. HV capacitors $(270 \mathrm{pF}$ ) decouple the fast signals from the high voltage. Surge arrester: ${ }^{1}$ are used to protect the readout electronics from discharges. In order to measure the signals, custom-made low-noise JFET charge preamplifiers with a integration time constant of $470 \mu$ s were developed with a design derived from Ref. 12. The charge integrator has four low noise BF862 FET transistors from Philips Semiconductor ${ }^{2}$ connected in parallel to match a high detector capacitance. Its charge sensitivity is $1 \mathrm{mV} / \mathrm{fC}$, as determined by the $1 \mathrm{pF}$ feedback capacitance. The integrator stage is followed by a RC-CR shaper with a gain of about 10 and shaper integration and differentiation time constants of $0.6 \mu$ s and $2 \mu$ s, respectively. The amplifiers were designed to be compatible with both positive and negative inputs and are provided with an input for the adjustment of the output baseline, in order to utilize the full dynamic range of the digitizer. Two preamplifier channels are housed on a single hybrid. We measured a sensitivity of about $12 \mathrm{mV} / \mathrm{fC}$ and a signal to noise ratio of 10 for $1 \mathrm{fC}$ input charge and $200 \mathrm{pF}$ input capacitance. The digitization and data acquisition is done by the CAEN SY2791 readout system 3 , specially developed as a complete readout system for LAr TPCs, housing up to 8 boards with 32 channels each, with one preamplifier per channel, $2.5 \mathrm{MHz}$ 12-bit flash ADCs, trigger logic, and an optical link.

\section{Electric fields configuration}

The electric field configuration is constrained by a few requirements: (a) the drift field is set to $500 \mathrm{~V} / \mathrm{cm}$, which corresponds to a drift velocity of $1.63 \mathrm{~mm} / \mu \mathrm{s}$ [13] and the electron-ion-pair recombination factor is $70 \%$ [14] for

\footnotetext{
${ }^{1}$ EC 90, EPCOS AG, Munich, Germany

${ }^{2}$ Now available at http://www.nxp.com.

${ }^{3}$ CAEN S.p.A., Viareggio, Italy, http://www.caen.it
} 

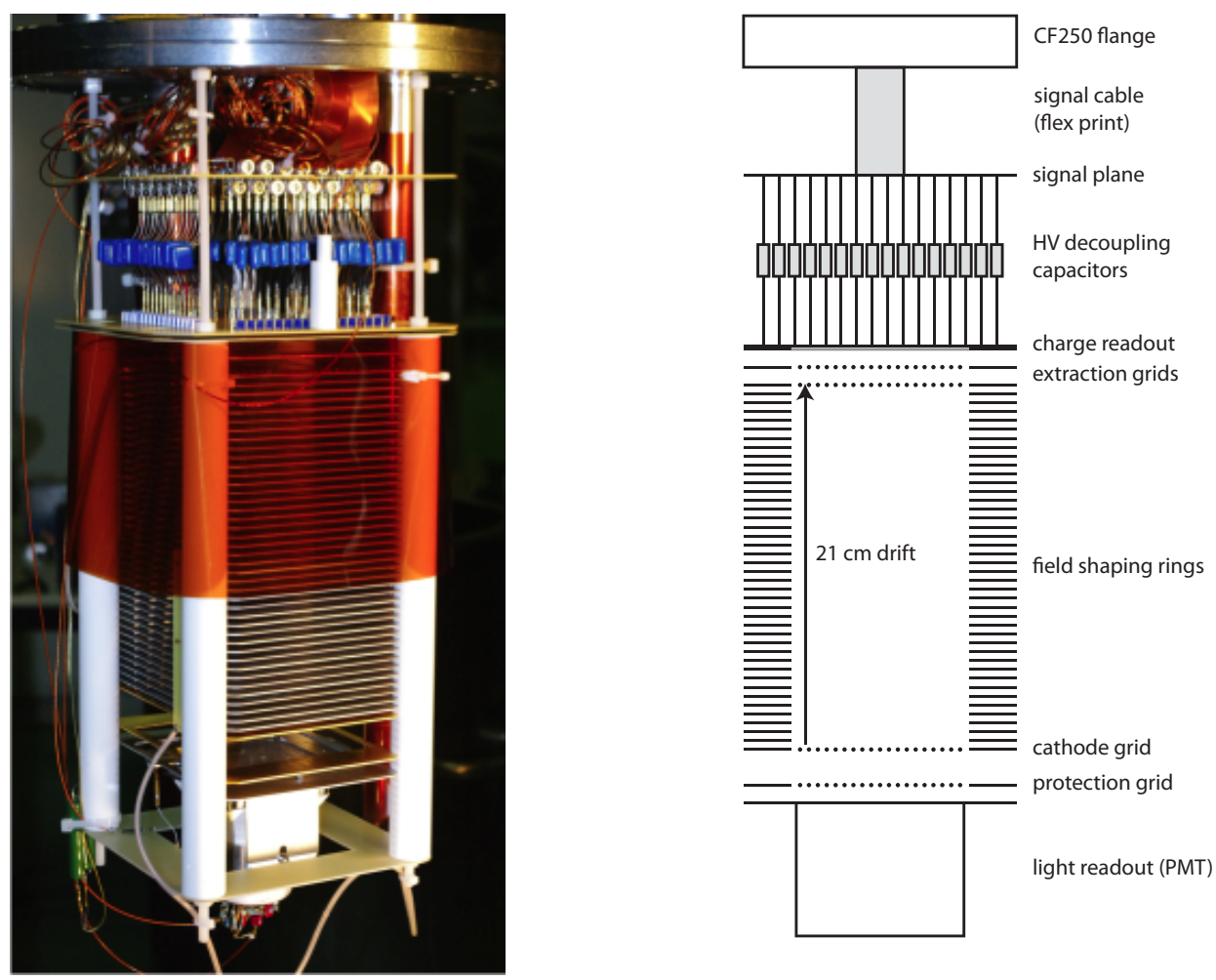

Figure 2: Left: picture of the 3L prototype LEM-TPC. Right: schematics of a LEM-TPC.

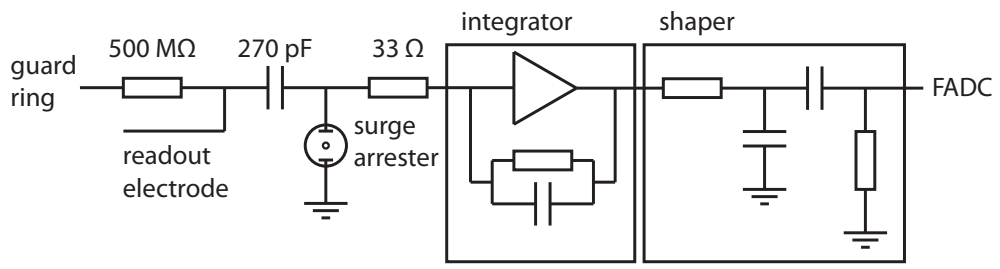

Figure 3: Electrical schematics of each readout channel of the anode. 
minimum ionizing tracks; (b) in order to extract electrons fast and efficiently from the liquid to the vapor phase, extraction fields larger than $2.5 \mathrm{kV} / \mathrm{cm}$ are applied [15, 16]; (c) the field inside the $1 \mathrm{~mm}$ thick LEM holes must reach $35.5 \mathrm{kV} / \mathrm{cm}$ (see section 4); (d) the strengths of the transfer fields must minimize the use of high voltages. With such fields, the charge produced in the liquid must be drifted, extracted and efficiently collected on the anode plane. The electric charges will traverse several regions with different electric fields. The transparency of the system drift-extraction grid-LEM-anode must be maximized by appropriate choice of the transfer fields. In addition, the amount of charge collected on the $\mathrm{X}$ - and $\mathrm{Y}$-views of the anode depends on their potentials and geometry. Therefore, the electric field configuration of the entire system has been numerically simulated and optimized, using COMSOL Multiphysic:4. The typical configurations of the electric fields are reported in Table 1. The charge is emitted into the gas phase by means of a $3 \mathrm{kV} / \mathrm{cm}$ extraction field. In order to maximize the LEM transparency, the field between the extraction grid in GAr and the bottom LEM electrode is reduced down to $1.5 \mathrm{kV} / \mathrm{cm}$ and the field between LEM and Anode is equal to $3 \mathrm{kV} / \mathrm{cm}$. The corresponding voltage applied across the two electrodes of the $1 \mathrm{~mm}$ thick LEM is as high as $3.55 \mathrm{kV}$. The two anode views are kept at the same potential and their geometries are defined to collect equal amounts of charge. Figure 4 shows the computed electric field lines. The effect of the field line squeezing in the holes is shown in the corresponding zoomed area. The impact of the extraction grids on the field lines is also visible. We note that because of the changing dielectric constant, the field above the liquid is $1.5 \times$ stronger than in the liquid.

\begin{tabular}{lrl}
\hline \hline Anode-LEM & $3 \mathrm{kV} / \mathrm{cm}$ & \\
LEM holes & $30-35.5 \mathrm{kV} / \mathrm{cm}$ & Table 1: Nominal configuration of elec- \\
Grid-LEM & $1.5 \mathrm{kV} / \mathrm{cm}$ & tric fields. \\
Extraction (in LAr) & $3 \mathrm{kV} / \mathrm{cm}$ & \\
Drift & $500 \mathrm{~V} / \mathrm{cm}$ & \\
\hline \hline
\end{tabular}

${ }^{4}$ COMSOL multiphysics, http://www. comsol.com 


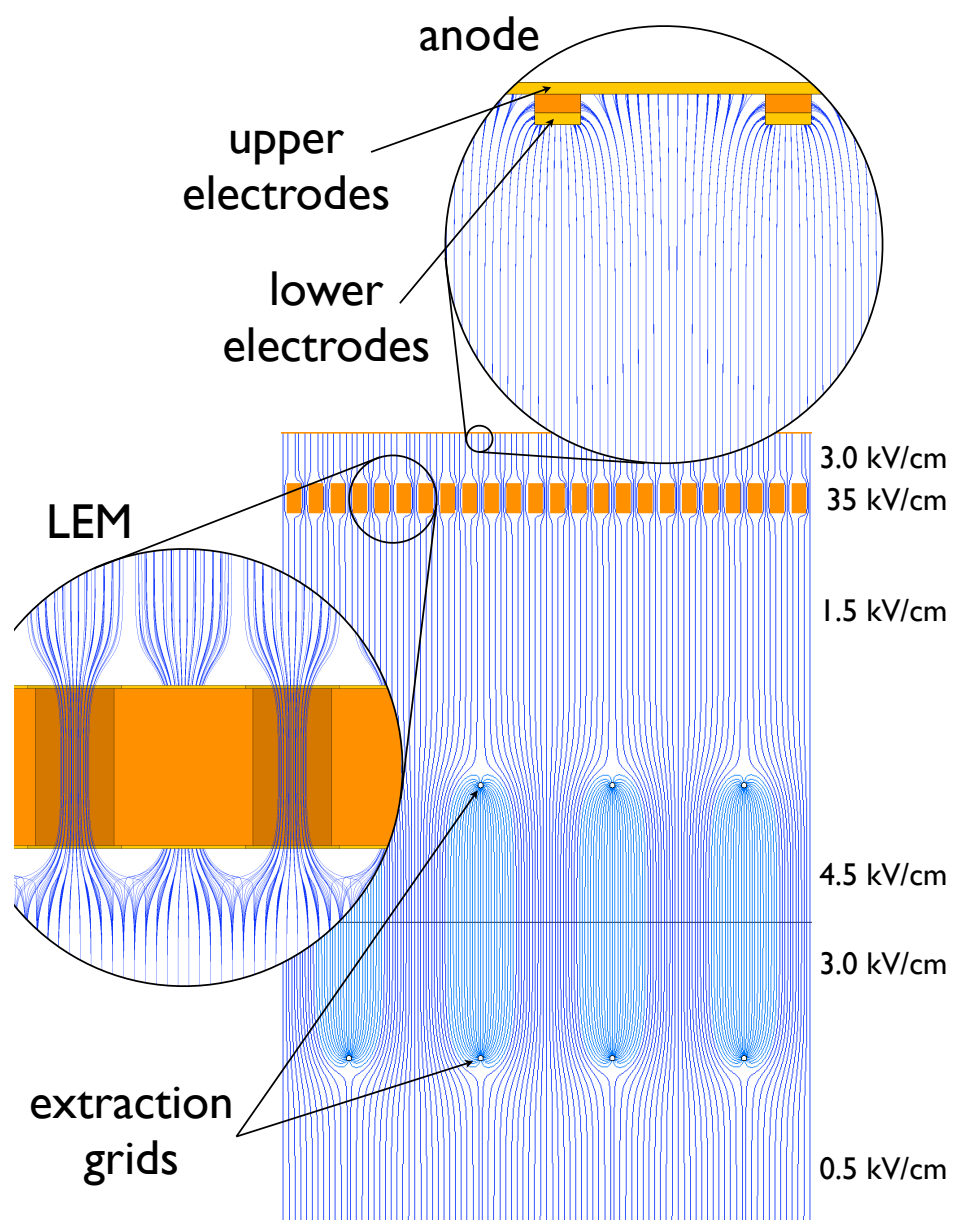

Figure 4: Overall electric field configuration including (a) drift region (b) extraction from liquid to vapor (c) drift towards LEM holes (d) LEM holes (e) drift towards anode (f) fields near the anode strips. The displayed configuration corresponds to equal charge sharing between $\mathrm{X}$ - and Y-views. 


\section{The Large Electron Multiplier (LEM)}

The general technique of electron multiplication via avalanches in small holes is attractive because (1) the required high electric field can be naturally attained inside the holes and (2) the finite size of the holes effectively ensures a confinement of the electron-ion pair avalanche, thereby reducing secondary effects in a medium without quencher. The gain $(G)$ in a given uniform electric field of a parallel plate chamber at a given pressure is described by $G \equiv e^{\alpha d}$ where $d$ is the gap thickness and $\alpha$ is the first Townsend coefficient, which represents the number of electrons created per unit path length by an electron in the amplification region. The behavior of this coefficient with density and electric field can be approximated by the Rose and Korff law [17]: $\alpha(E)=A \rho e^{-B \rho / E}$ where $E$ is the electric field, $\rho$ is the gas density, $A$ and $B$ are the parameters depending on the gas. A fit to the electric field dependence of the Townsend coefficient in the range between 20 and $40 \mathrm{kV} / \mathrm{cm}$ predicted by MAGBOLTZ [18] calculations, gives $A \rho=(3160 \pm 90) \mathrm{cm}^{-1}$ and $B \rho=$ $(136.4 \pm 1.0) \mathrm{kV} / \mathrm{cm}$ for pure argon at $87 \mathrm{~K}$ and 1 bar.

For amplification in holes, it is convenient to make use of the same formalism by replacing the gap thickness $d$ by the effective amplification path length within the holes, called $x$, which can be related to the length of the field plateau along the hole. The effective gain is then expressed as:

$$
\left.G_{\text {eff }} \equiv T \exp \left[A \rho x e^{\left(-B \rho /\left(\kappa E_{0}\right)\right.}\right)\right]
$$

where $A \rho, B \rho$ are given by MAGBOLTZ, $E_{0}=V / d$ is the nominal electric field and $d$ the LEM thickness, $\kappa E_{0}$ the effective field, and $T$ is the transparency (some electrons, in part due to the large diffusion in pure argon, may be collected on the grids or on the electrodes of the LEM). Electrostatic calculations of the LEM-hole geometry give a maximum field in the hole which is lower than the naive $V / d$, consistent with a value of $\kappa=0.95$ and an effective length in the range of $0.7 \mathrm{~mm}$ for a $1 \mathrm{~mm}$ thick LEM.

Electron multiplication in holes has been experimentally investigated for a large number of applications. The most extensively studied device is the Gas Electron Multiplier (GEM) [7], made of 50-70 $\mu \mathrm{m}$ diameter holes etched in a $50 \mu \mathrm{m}$ thick metallized Kapton foil. Stable operation has been shown with various gas mixtures and very high gains. An important step was the operation of the GEM in pure Ar at normal pressure and temperature [19]. Rather high gains were obtained, of the order of 1000, supporting evidence for the avalanche confinement to the GEM micro-holes. Operation of GEMs 
in an avalanche mode in pure Ar in double phase conditions has been studied [20], using triple-stage GEMs reaching gains of the order of 5000 .

The successes of the GEMs triggered the concept of the Large Electron Multiplier (LEM) also called thick GEM (THGEM) (for a review see [21]), a coarser but more rigid structure made with sub-millimeter-size holes in a millimeter-thick printed circuit board (PCB). Compared to GEMs, LEMs are mechanically more robust, are sturdy to withstand cryogenic temperatures and stresses, and have a good discharge hardness.

We have built several LEM prototypes using standard PCB techniques from different manufacturers. Double-sided copper-clad (of 16-50 $\mu \mathrm{m}$ layers) FR4 plates with thicknesses ranging from $0.8 \mathrm{~mm}$ to $1.6 \mathrm{~mm}$ were drilled with a regular pattern of $500 \mu \mathrm{m}$ diameter holes at a relative distance of $800 \mu \mathrm{m}$. Table 2 shows the parameters of the $1 \mathrm{~mm}$ thick LEMs used for this test and a picture of one such LEM is shown in Figure 5. The LEMs were manufactured at the CERN TS/DEM workshop with a specific technique to ensure alignment between holes and rims around the holes: the holes are Computer Numerical Control (CNC) drilled and dielectric rims are etched uniformly around the holes [22]. The copper layer thickness before etching was about $50 \mu \mathrm{m}$ and it reduced to about $30 \mu \mathrm{m}$ after rim etching. The resulting thickness of the copper layer is sufficient in order to have resistance to sparks. Thanks to this manufacturing technique, the dielectric rims are perfectly aligned with the holes (see Figure 6). Since the dielectric rim reduces the probability of a discharge [23] higher voltages can be applied between the two LEM electrodes. This increases the maximum gain that can be reached before a breakdown occurs.

\begin{tabular}{lr}
\hline \hline size & $10 \times 10 \mathrm{~cm}^{2}$ \\
PCB thickness & $1 \mathrm{~mm}$ \\
copper layer thickness (before etching) & $50 \mu \mathrm{m}$ \\
copper layer thickness (after etching) & $\approx 30 \mu \mathrm{m}$ \\
hole diameter & $500 \mu \mathrm{m}$ \\
hole pitch & $800 \mu \mathrm{m}$ \\
dielectric rim size & $50-60 \mu \mathrm{m}$ \\
\hline \hline
\end{tabular}

Table 2: Physical parameters of the Large Electron Multiplier (LEM). 


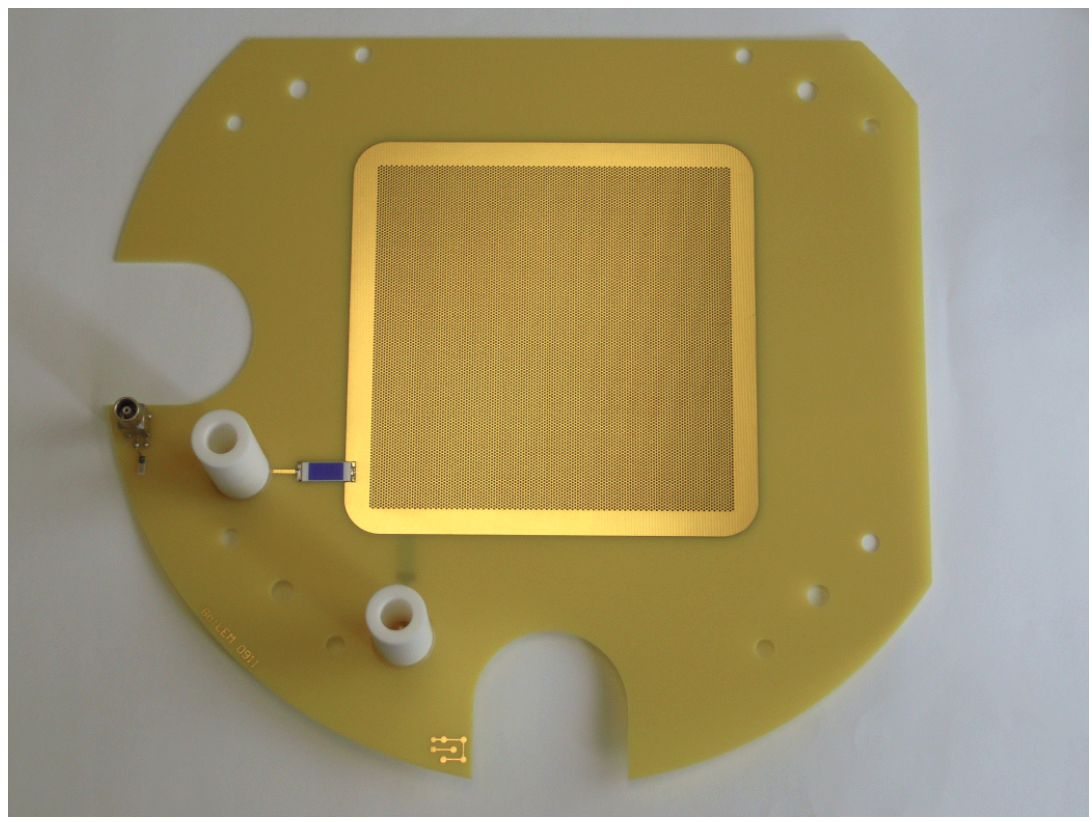

Figure 5: Picture of a $1 \mathrm{~mm}$ thick LEM with two Macor insulated HV connections and a $500 \mathrm{M} \Omega$ resistor.

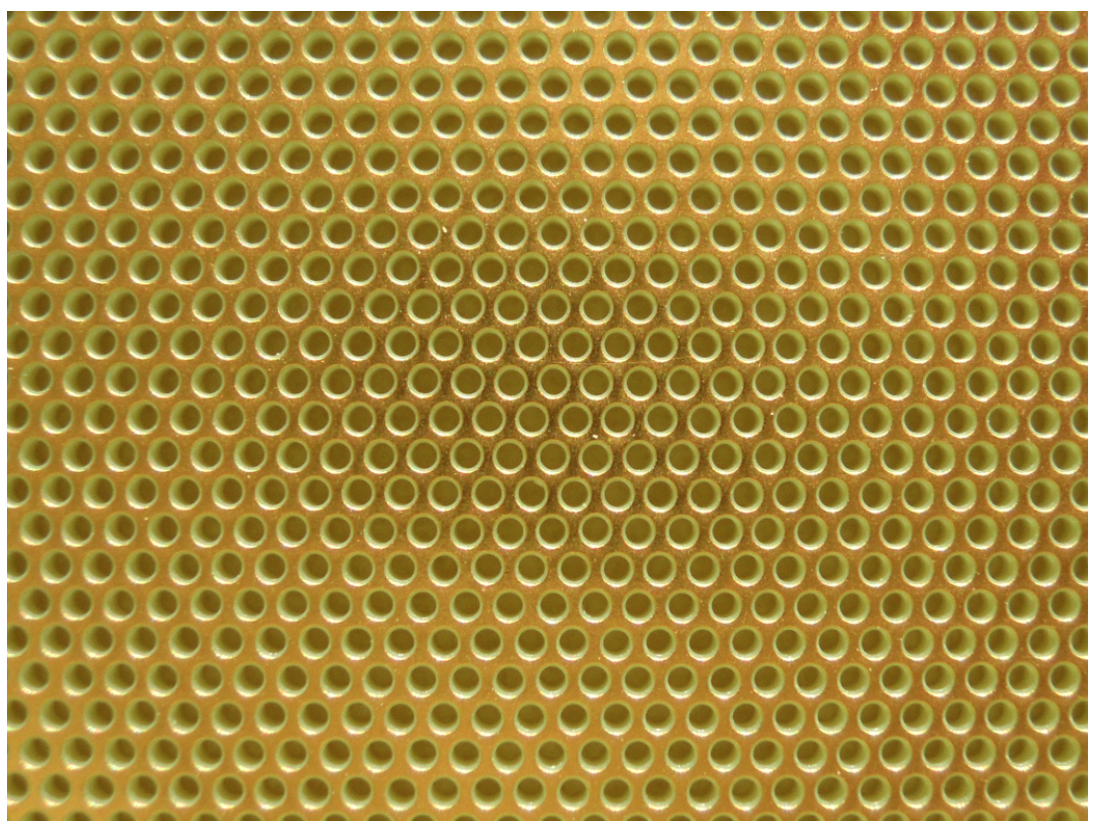

Figure 6: Close up of the LEM showing the well centered and uniform dielectric rims. 


\section{The projective $2 \mathrm{D}$ anode}

The $2 \mathrm{D}$ anode provides $\mathrm{X}$ - and Y-views, i.e. two independent planes with mm-scale readout pitch. It is based on the GEM 2D readout concept [9] and is conceptually shown in Figure 7. It is composed of two independent sets of strips oriented perpendicularly to each other, called "lower" and "upper" electrodes, and separated by a thin insulating film. Both sets of electrodes are operated at the same electric potential, and the geometry of their strips is chosen such that each electrode collects half of the charge. We note that the strips of the upper electrode are wider to compensate the screening of the thin strips of the lower electrode.

A prototype projective anode used for this test with the parameters described in Table 3 was produced by the CERN TS/DEM workshop, using photolithographic etching techniques. Pictures are shown in Figure 8. To produce the anode, one of the two copper surfaces of an insulating $50 \mu \mathrm{m}$ thick polyimide foil was etched with the pattern of the strips of the upper electrode and glued on a PCB support. The other copper surface was masked with the pattern of the lower electrode, first the copper and afterwards the polyimide were etched away leaving the strips of the upper electrode exposed. A fine strip pitch of $600 \mu \mathrm{m}$ ensures that charge clouds always induce signals on more than one strip of the electrodes. The final readout pitch of $3 \mathrm{~mm}$ is reached by electrically bridging 5 strips, as can be seen in Figure 8 (bottom).

\begin{tabular}{lr}
\hline \hline readout pitch & $3 \mathrm{~mm}$ \\
strip pitch & $600 \mu \mathrm{m}$ \\
lower electrode width & $120 \mu \mathrm{m}$ \\
upper electrode width & $500 \mu \mathrm{m}$ \\
kapton thickness & $50 \mu \mathrm{m}$ \\
\hline \hline
\end{tabular}

Table 3: Design parameters of the projective 2D anode.

\section{Operation in double phase liquid Argon}

In order to be able to measure tracks in the whole active volume, it is necessary that the mean lifetime of the drifting free electrons in LAr is at least of the order of the maximal drift time. A drift of $21 \mathrm{~cm}$ with an electric field of $500 \mathrm{~V} / \mathrm{cm}$ without significant charge loss requires the LAr to contain less than few ppb of electronegative impurities like $\mathrm{O}_{2}, \mathrm{H}_{2} \mathrm{O}$ and $\mathrm{CO}_{2}$. This 


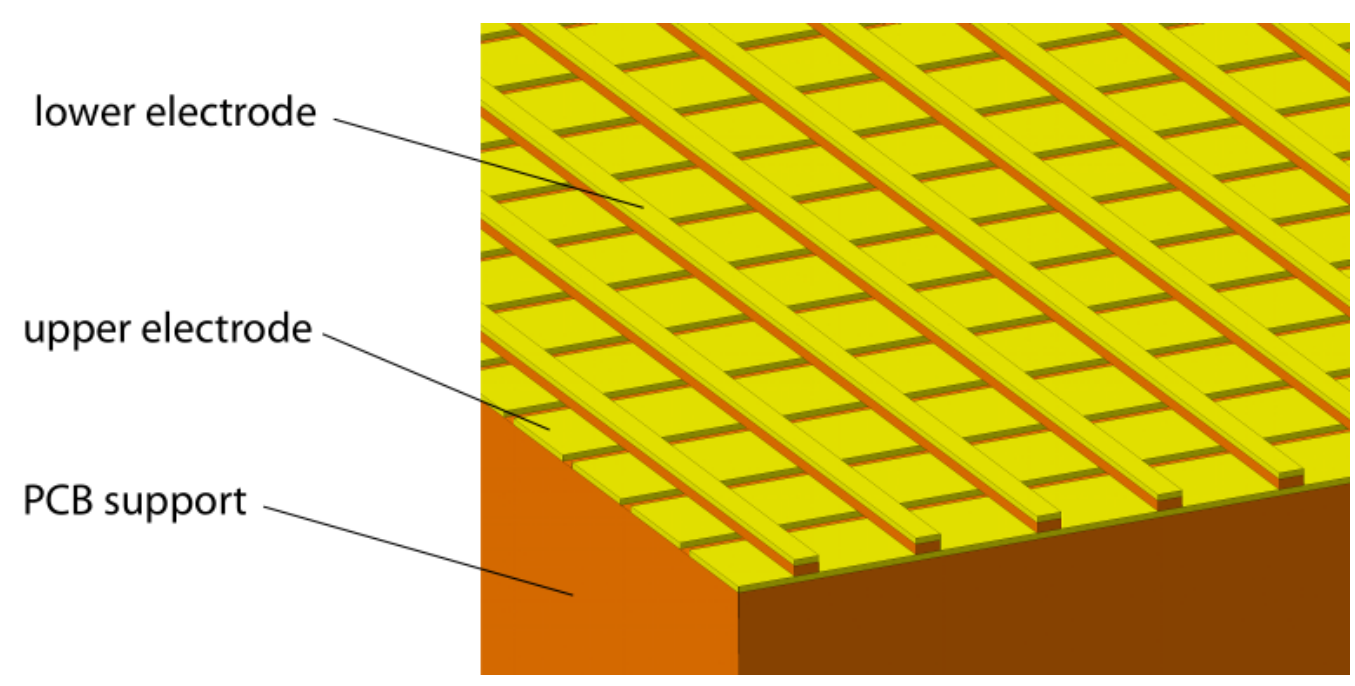

Figure 7: Illustration of the projective 2D anode. See text.

makes it necessary to purify the commercial grade LAr (declared content of $\mathrm{O}_{2}<5 \mathrm{ppm}$ and $\mathrm{H}_{2} \mathrm{O}<10 \mathrm{ppm}$ ) used to fill the detector.

Precautions are used to ensure a good purity of the argon during operation: after the assembly, the detector, the recirculation system and the cartridges are pumped down to $<10^{-6}$ mbar. To speed up the cleaning we warm the detector vessel from outside without exceeding $50^{\circ} \mathrm{C}$, mainly not to damage the photocathode of the PMT.

An external cooling bath is filled with LAr in order to cool the detector down to $87 \mathrm{~K}$. After it reaches the final temperature, the inner vessel is filled with argon. During the filling, argon is fed into a custom-made filter, filled with pure copper powder. The copper oxidizes trapping oxygen impurities from the liquid; this cartridge can be regenerated by increasing the temperature and flushing argon-hydrogen mixture to induce $\mathrm{CuO}_{2}+2 \mathrm{H}_{2} \rightarrow \mathrm{Cu}+2 \mathrm{H}_{2} \mathrm{O}$.

To maintain the purity for long periods the liquid argon is evaporated and pushed by a metal bellows pump through a commercial SAES getter ${ }^{5}$. (See Ref. [6] for details on the purification system). The cleaned gas argon is recondensed into the detector by means of a spiral condenser. The argon flow is regulated via a needle valve at the input of the pump and it is monitored with a mass flow meter mounted between the pump and the getter. We

\footnotetext{
${ }^{5}$ SAES Pure Gas Inc., MicroTorr MC400.
} 

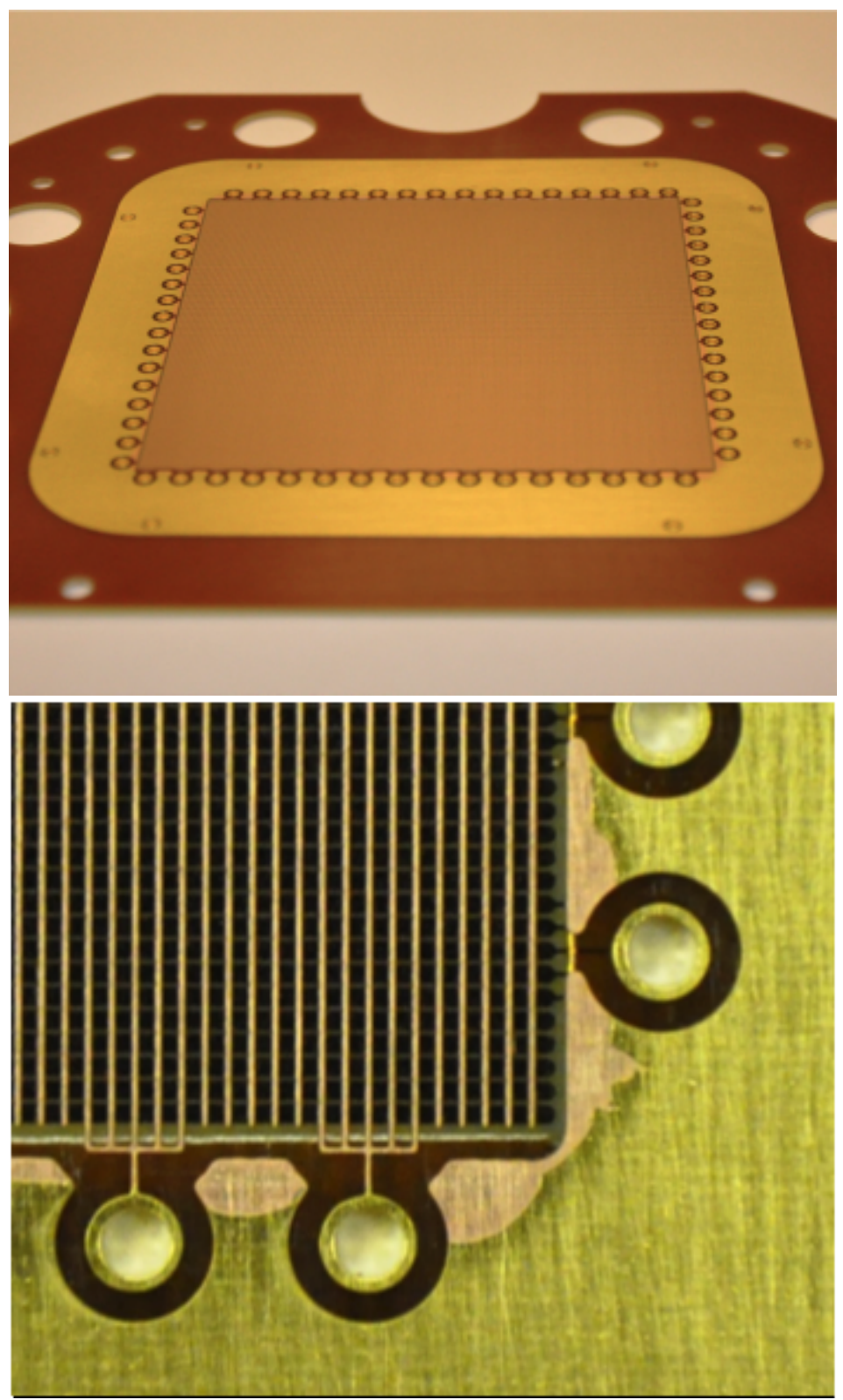

Figure 8: Pictures of the manufactured projective 2D anode prototype. (top) overall view (the readout area is $10 \times 10 \mathrm{~cm}^{2}$ ). (bottom) zoom on the strips showing the bridging of 5 strips to obtain a $3 \mathrm{~mm}$ readout pitch. 
operate the recirculation at $7.5 \mathrm{slm}$ : two days are needed for a full volume change.

Moreover the argon filling procedure is crucial: the recirculation system is turned on while cooling the detector. This procedure has two advantages: the outgassed molecules are trapped into the getter cartridge during the recirculation and the presence of argon gas inside the detector favors uniform and fast cooling of all the the parts.

The free electron lifetime measurement which was done about one day after the filling indicated an initial purity of about $1 \mathrm{ppb}$. The corresponding plot with the description of the analysis is shown in the next section. After the filling the detector has been operated during one week without any argon purification.
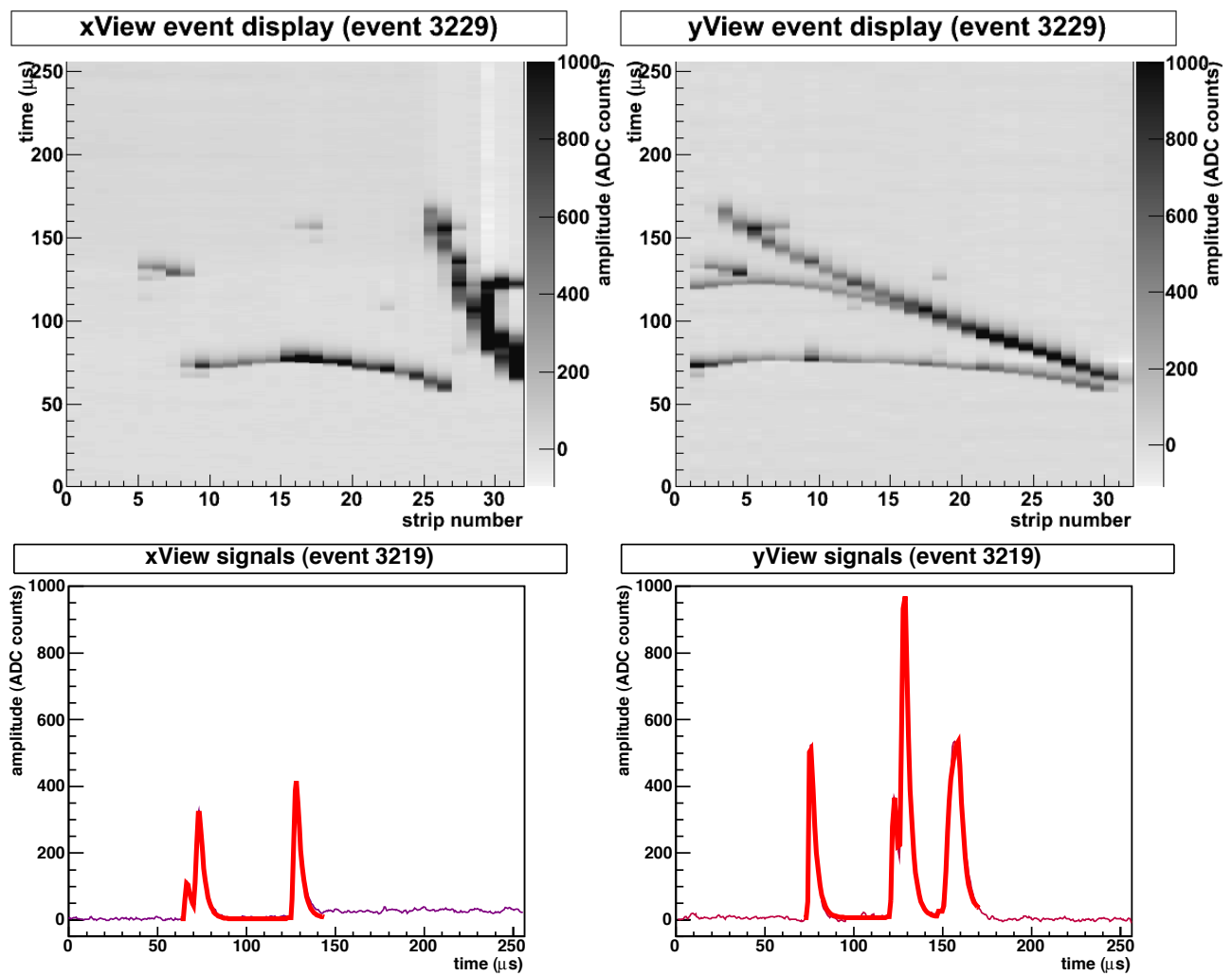

Figure 9: Multi-track event. Top: event display: channel number vs. drift time, x-view left, and y-view right. The grey scale is proportional to the signal amplitude. Bottom: Typical waveforms for the $\mathrm{x}$-view left and the $\mathrm{y}$-view right. The thick red curve shows the result of the signal fit using the response of the preamplifier. 

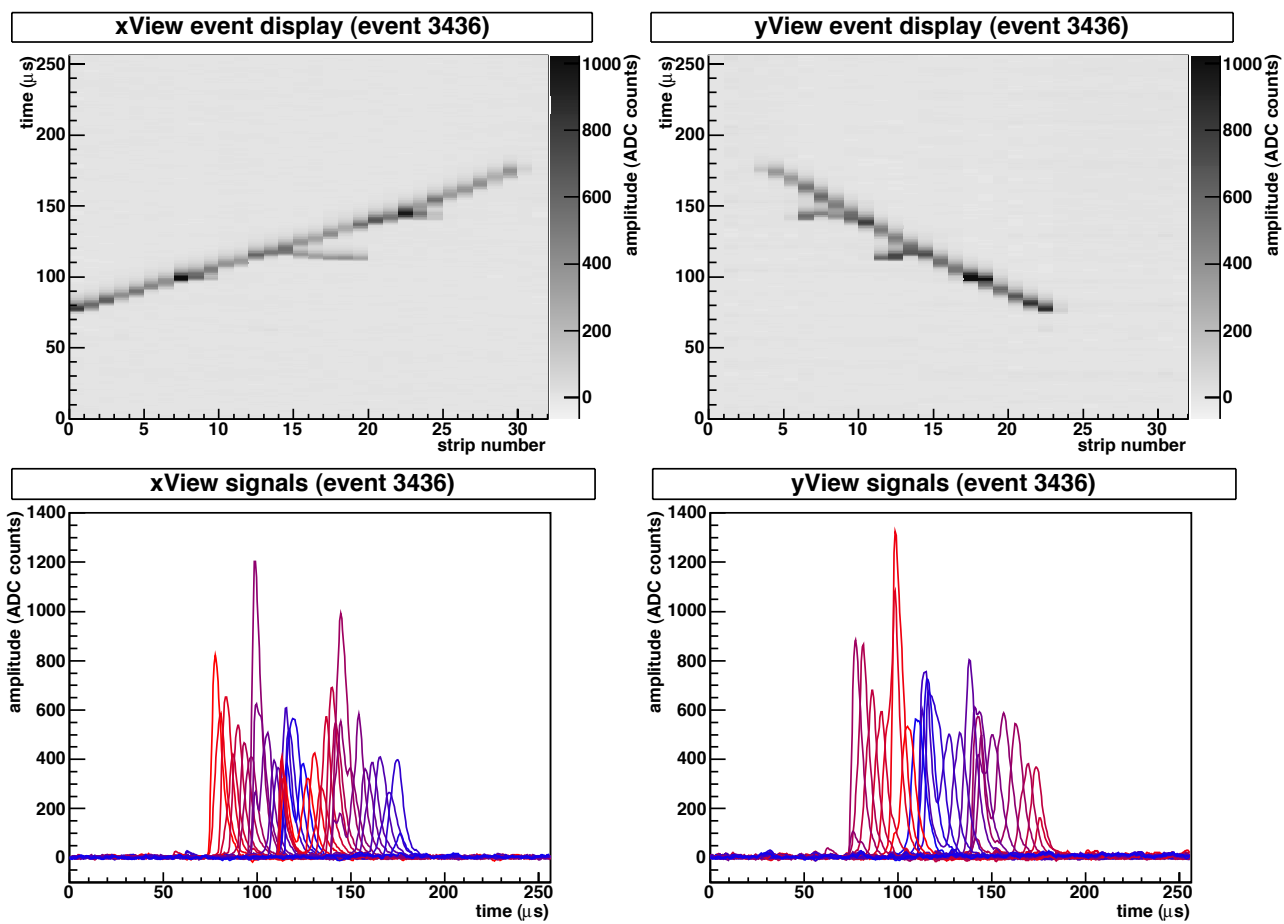

Figure 10: Muon crossing the chamber with visible delta rays. Top: event display: channel number vs. drift time, $\mathrm{x}$-view left, and y-view right. The grey scale is proportional to the signal amplitude. Bottom: 32 waveforms of the $\mathrm{x}$-view left and the $\mathrm{y}$-view right.

\section{Muon track reconstruction and free electron lifetime correction}

The aim of this section is to describe the reconstruction procedure which was used to select and evaluate long tracks.

Because the ground configuration of this setup was not fully optimized, coherent noise was observed on all channels. After applying a mean trace subtraction of channels without a signal to remove such coherent noise, waveforms are very clean. Figure 9 shows a collected multi-track event after the digital filter. Hits are discriminated from the residual noise by a hit finding algorithm. Found hits fit the well-known response function of the preamplifier convoluted with a constant current at its input, whose length is left as a free parameter. Typical fit results are shown with thick curves on the waveform plots.

After a calibration with charge pulses the signal integral is converted into the charge collected on the strip. Besides the charge of a hit, the start time 

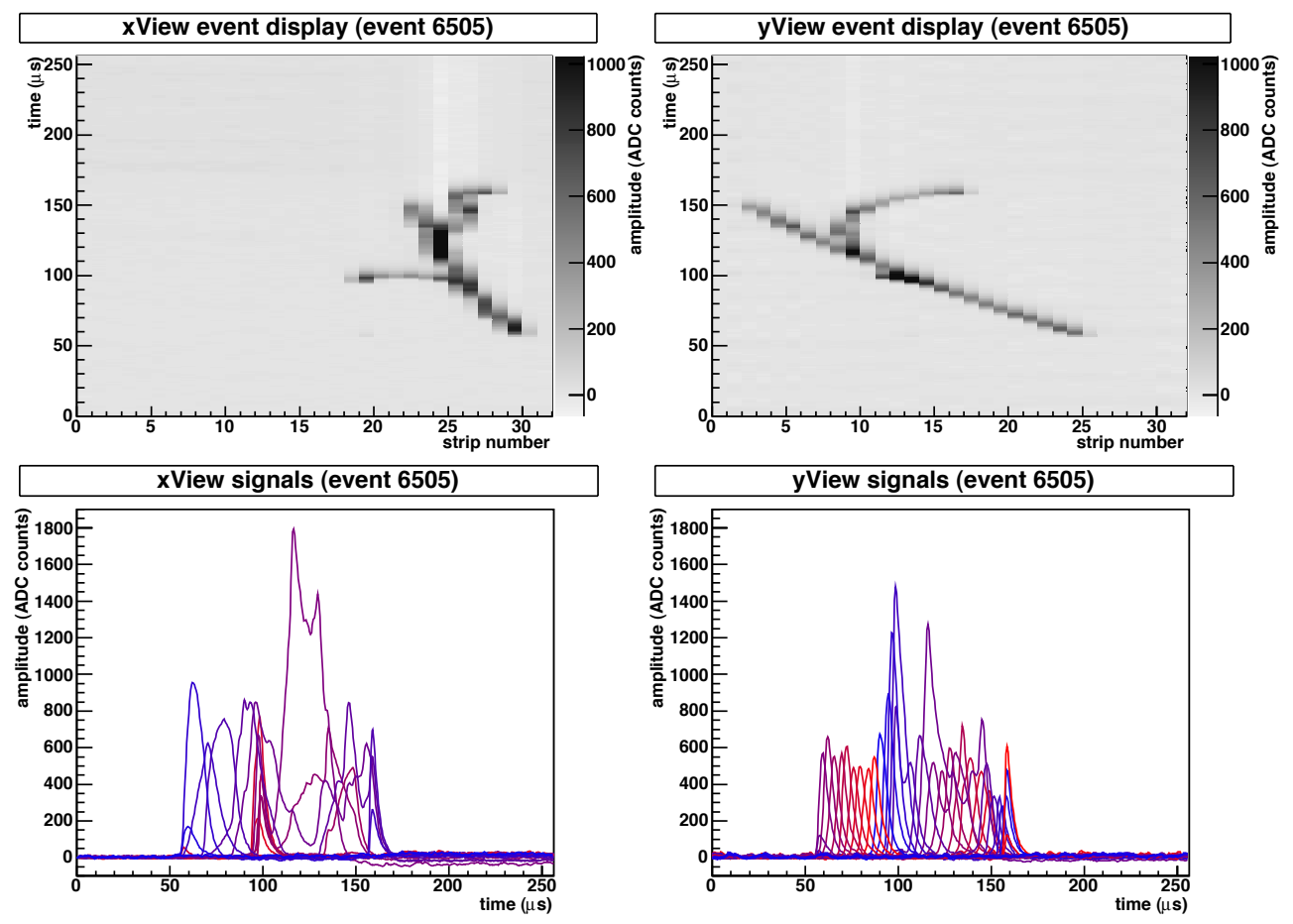

Figure 11: ibid, see caption of Fig. 10

of the signal is also extracted from the fit. The drift coordinate $z$ of the hit is then given by the electron drift velocity multiplied by the time difference between the trigger signal from the PMT and the start time of the signal. The strip number gives the coordinate of the corresponding view.

Figure 10 and 11 show two ionizing events with long tracks, triggered by the primary scintillation light which was detected with the PMT. After clustering of connected hits, matching 2D tracks were fitted with the linear functions $z(x)$ and $z(y)$ which could then be solved to extract the missing coordinates ( $\mathrm{y}$ of $\mathrm{X}$-view and $\mathrm{x}$ of $\mathrm{Y}$-view). Eventually long tracks crossing the full active volume were selected based on cuts on the endpoints of the track and also the goodness of the linear fits.

The energy loss of the long muon tracks was used to estimate the effective gain of the chamber. The $d Q / d x$ value of each hit is defined by the ratio of the hit integral and the track length below the considered strip. After dividing the active volume into slices of equal drift length, a Gauss convoluted Landau function was fitted to each of the obtained collected charge per unit length 


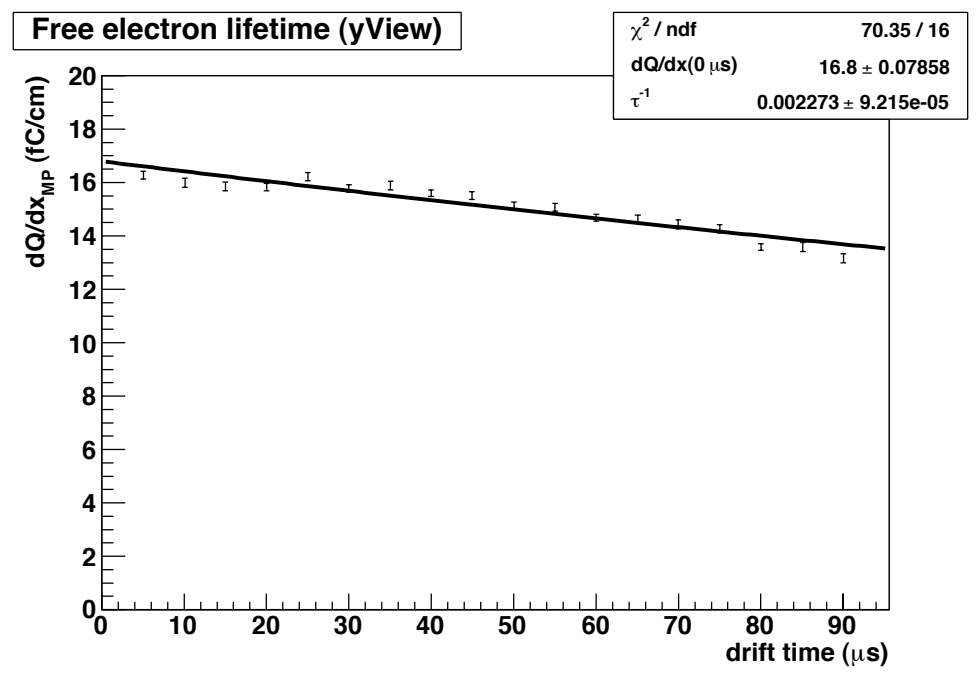

Figure 12: Most-probable stopping power $(d Q / d x)_{\mathrm{M} P}$ in $\mathrm{fC} / \mathrm{cm}$ for different slices of drift time. The curve is an exponential fit characterizing the free electron lifetime. The error bars show the statistical error of the Landau fit (see text). For this data, the LEM gain was about 3 .

distributions. By plotting the most-probable values of these distributions against the drift time, the free electron lifetime can be obtained by fitting an exponential decay to the points. See Figure 12. The error bars show the statistical error of the Gauss-convoluted Landau fit. The measured free electron lifetime is $(440 \pm 20) \mu \mathrm{s}$, which corresponds to a concentration of about $(0.68 \pm 0.03)$ ppb of oxygen equivalent impurities in LAr [24].

\section{Signal amplification and effective gain}

The signal induction on the two views is based on the principle of charge sharing between them. In order to get a quantitative measurement, long muon tracks, selected as described in the previous section, were used. The left plot of Figure 13 shows a scatter plot of the total amount of charge collected on the X-vs the Y-view. As shown in the histogram on the right side of the Figure the normalized difference of the charge collected on the two views is better than $5 \%$.

The second issue which has been addressed is the amplification of the system. The effective gain is defined as the sum of the collected charge per unit length on the two anode views (corrected for the drifting electron lifetime) 

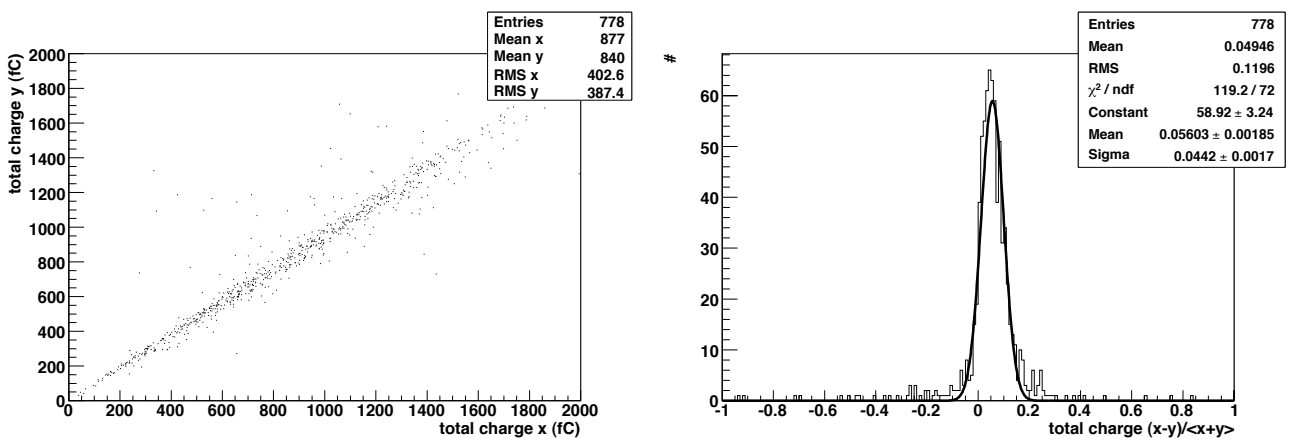

Figure 13: Left: Scatter plot of total charge collected on the $\mathrm{x}$-view versus the charge collected on the y-view. Right: histogram of the charge collection difference, normalized by the average charge.

divided by the charge per unit length released in liquid argon by a minimum ionizing muon at the given electric field. The former is estimated from the $d Q / d x$ distribution of the reconstructed muon tracks. Minimum ionizing particles (mip) deposit in LAr an energy of $1.519 \mathrm{MeV} \mathrm{cm}^{2} / \mathrm{g}$. For our drift field configuration of $500 \mathrm{~V} / \mathrm{cm}$ and taking into account $30 \%$ recombination, a total charge of $10 \mathrm{fC} / \mathrm{cm}$ is released for mip tracks.

The effective gain, in addition to the charge multiplication in the LEM holes, takes into account potential charge losses from the efficiency to extract the electrons to the vapor phase and from the transparency of the grids and the LEM. For this reason the effective gain is sensitive to all the details of the electric field configuration. The working point is found from electrostatic computations of the drift lines (see Section 3) and from the optimization of the transparency.

In order to measure the gain as a function of the amplification field the voltage across the LEM electrodes was increased from $3000 \mathrm{~V}$ up to $3550 \mathrm{~V}$, where first discharges induced by ionizing events occurred, while leaving the other fields constant. Figure 14 shows the normalized $d Q / d x$ distributions of long cosmic muon tracks measured with one of the two views. All the distributions were fitted with a Gauss convoluted Landau function. Figure 15 shows the effective gain as a function of the applied voltage.

The maximum effective gain obtained in stable conditions is 27 , with a signal to noise ratio larger than 200 for minimum ionizing particles. The measured effective gains as a function of the applied voltage can be compared to the predictions, as presented in Section 4. The gain curve fits the 
functional form in Eq. 1 and one determines the effective thickness $x$ and the effective field parameter $\kappa$. Figure 16 shows the fitted values as a function of the assumed transparency $T$. It demonstrates that the observed gain curve is consistent with expectations. If we assume a value $\sim 50 \%$ for the transparency, the data point to $x=0.7 \mathrm{~mm}$, and $\kappa=0.96$. These values are perfectly compatible with the expectations from electrostatic field calculations.

We can also address the charge measurement resolution and its potential degradation due to fluctuations of the amplification in the holes. In order to compare the different situations, we study the $(d Q / d x) / G$ distributions, i.e. the measured $d Q / d x$ divided by the gain $\mathrm{G}$, obtained with the reconstructed cosmic muon tracks. The histograms, with the corresponding fits, for different LEM fields are overlapped in Figure 17. The widths, characterized by the Landau distribution of the medium and the resolution, are similar for all gains, showing that the amplification stage does not degrade the observed $d E / d x$ distribution.

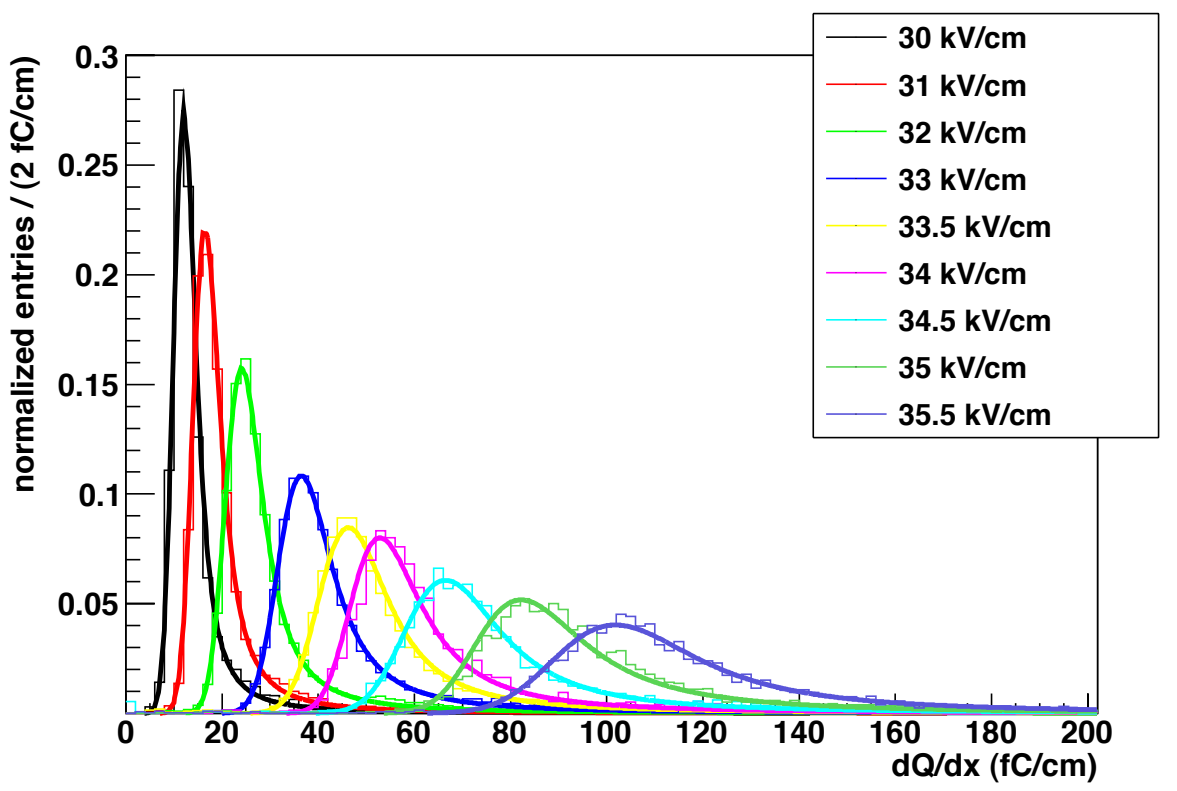

Figure 14: $d Q / d x$ distributions of reconstructed cosmic muon tracks with different LEM nominal fields. A Gauss convoluted Landau Function is fitted to each distribution. 


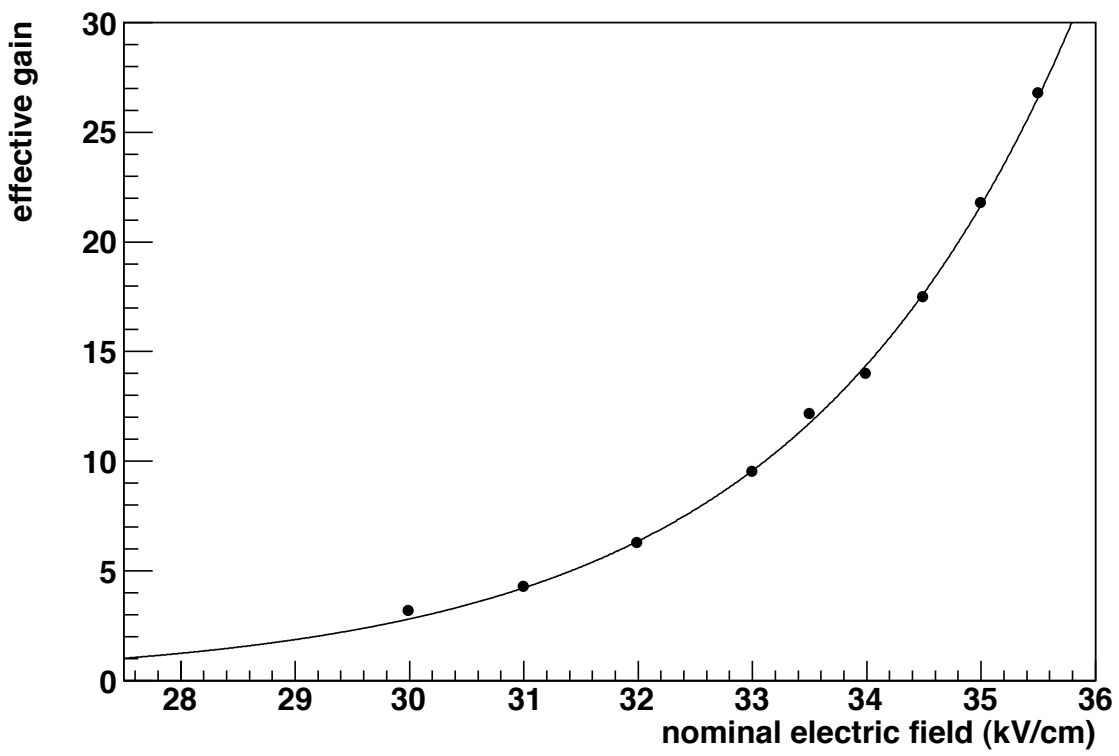

Figure 15: Effective gain as a function of the nominal electric field in the LEM holes. The curve is a model fit to the measured gain. See text.

\section{Conclusion}

The LAr LEM-TPC is a novel kind of complete calorimetric and tracking detector capable of charge multiplication. For the first time we operated a detector based on a decoupled LEM amplification stage, that allows adjustable charge amplification in argon vapor, and a 2D projective readout.

From the collected set of cosmic muon tracks, we observe the considerable improvement in the signal to noise ratio obtained thanks to the gain, opening the path to very long drift liquid Argon TPCs. The achieved stable gain of 27 can compensate for e.g. the attenuation of the collected charge for drifts of the order of $10 \mathrm{~m}$ even in the presence of diffusion or charge attenuation from impurities. For direct Dark Matter search with imaging, higher gains $(\gtrsim 100)$ are needed to reach an energy threshold down to tens of $\mathrm{keV}$ for nuclear recoil detection.

This proof of principle therefore represents another important milestone in the realization of very large, long drift (cost-effective) LAr detectors for next generation neutrino physics and proton decay experiments, as well as for direct search of Dark Matter with imaging devices. We believe that this 


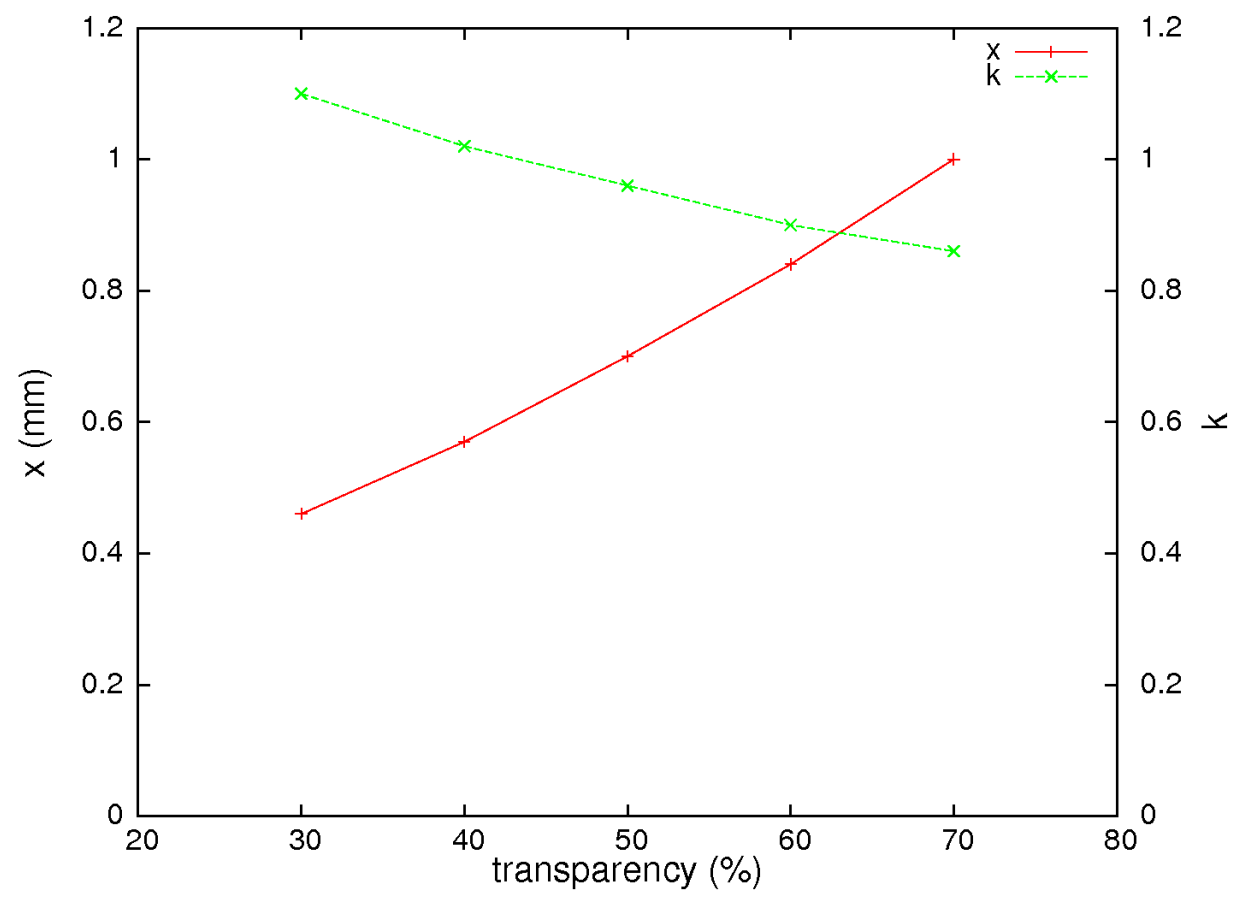

Figure 16: Fitted effective thickness $x$ and the effective field parameter $\kappa$ as a function of transparency $T$. See text.

technology is scalable up to very large detectors, for instance instrumenting the surface with a collection of $\sim 1 \times 1 \mathrm{~m}^{2}$ independently operating units.

\section{Acknowledgements}

This work was supported by ETH Zürich and the Swiss National Science Foundation (SNF). We are grateful to CERN for their hospitality and thank R. Oliveira and the TS/DEM group, where several of the components our of detector were manufactured. We also thank the RD51 Collaboration for useful discussions and suggestions.

\section{References}

[1] A. Badertscher et al., "Construction and operation of a Double Phase LAr Large Electron Multiplier Time Projection Chamber," arXiv:0811.3384 [physics.ins-det]]. 


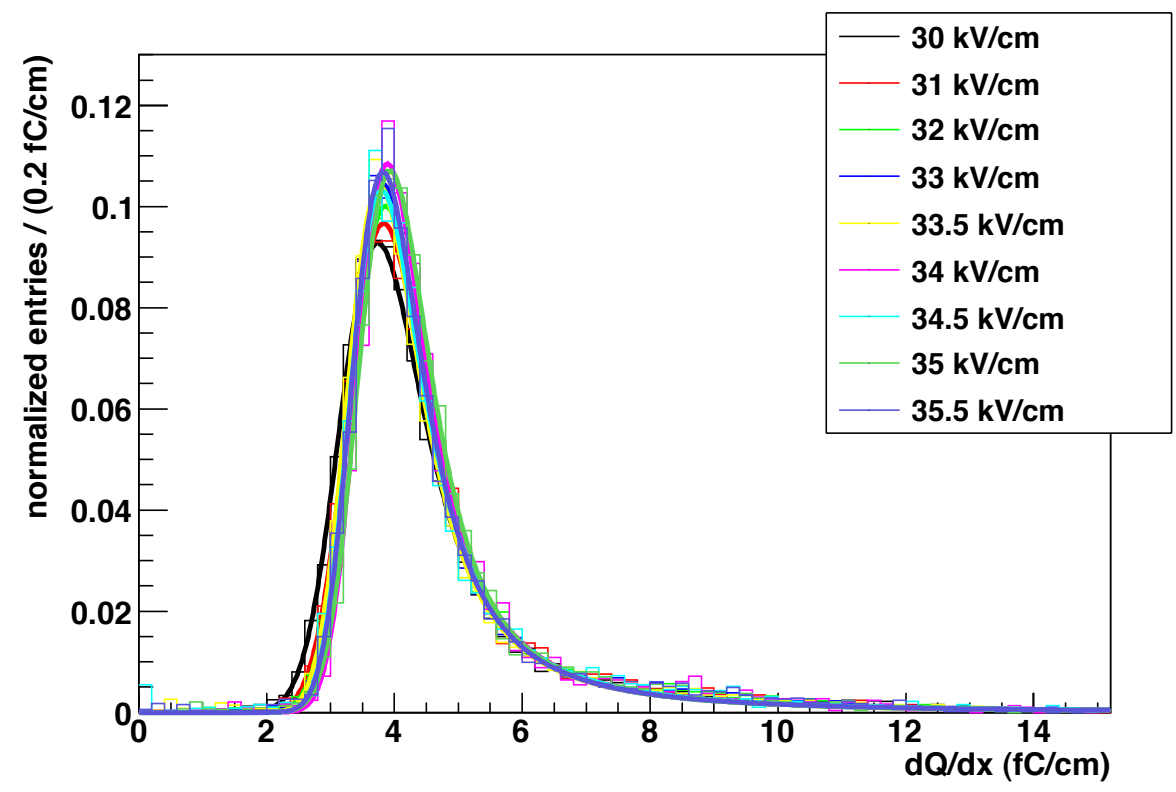

Figure 17: Distribution of the gain normalized $d Q / d x / G(\mathrm{fC} / \mathrm{cm})$ of reconstructed tracks, for different LEM nominal fields. In order to compare the distribution measured at different gains, we divided the $d Q / d x$ entries by the expected gain. The measured width of the Landau distribution, characteristic of the medium, is unaffected by the gain.

[2] A. Rubbia, "Experiments for CP violation: A Giant liquid argon scintillation, Cerenkov and charge imaging experiment?," [hep-ph/0402110].

[3] A. Rubbia, "Underground Neutrino Detectors for Particle and Astroparticle Science: The Giant Liquid Argon Charge Imaging ExpeRiment (GLACIER)," J. Phys. Conf. Ser. 171, 012020 (2009). arXiv:0908.1286 [hep-ph]].

[4] A. Badertscher et al., "Giant Liquid Argon Observatory for Proton Decay, Neutrino Astrophysics and CP-violation in the Lepton Sector (GLACIER)," arXiv:1001.0076 [physics.ins-det]].

[5] A. Rubbia, "ArDM: A Ton-scale liquid Argon experiment for direct detection of dark matter in the universe," J. Phys. Conf. Ser. 39, 129-132 (2006). [hep-ph/0510320].

[6] A. Badertscher et al., "Operation of a double-phase pure argon Large 
Electron Multiplier Time Projection Chamber: Comparison of single and double phase operation," Nucl. Instrum. Meth. A617, 188-192 (2010). arXiv:0907.2944 [physics.ins-det]].

[7] F. Sauli, "GEM: A new concept for electron amplification in gas detectors," Nucl. Instrum. Meth. A386, 531-534 (1997).

[8] A. Badertscher et al., "Stable operation with gain of a double phase Liquid Argon LEM-TPC with a $1 \mathrm{~mm}$ thick segmented LEM," arXiv:1010.2482 [physics.ins-det]].

[9] A. Bressan et al., "Two-dimensional readout of GEM detectors," Nucl. Instrum. Meth. A425, 254-261 (1999).

[10] V. Boccone et al. [ The ArDM Collaboration ], "Development of wavelength shifter coated reflectors for the ArDM argon dark matter detector," JINST 4, P06001 (2009). arXiv:0904.0246 [physics.ins-det]].

[11] T. Takahashi et al., Nucl. Instrum. and Meth. in Physics Research 205, 591-596 (1983).

[12] C.Boiano, R.Bassini,A.Pullia. and A.Pagano, "Wide-dynamic-range fast preamplifier for pulse shape analysis of signals from high-capacitance detectors", IEEE Transactions on Nuclear Science 51 1931-1935 (2004).

[13] W. Walkowiak, "Drift velocity of free electrons in liquid argon," Nucl. Instrum. Meth. A449, 288-294 (2000).

[14] S. Amoruso et al. [ ICARUS Collaboration ], "Study of electron recombination in liquid argon with the ICARUS TPC," Nucl. Instrum. Meth. A523, 275-286 (2004).

[15] E. M. Gushchin, A. A. Kruglov and I. M. Obodovski, "Emission of hot electrons from liquid and solid argon and xenon", Sov.Phys, JETP 55 (1982).

[16] A. F. Borghesani and G. Carugno and M. Cavenago and E. Conti, "Electron transmission through the Ar liquid-vapor interface", Physics Letters A149, 481-484 (1990).

[17] T. Aoyama, "Generalized Gas Gain Formula for Proportional Counters", Nucl. Instr. and Meth. A 234 (1985) 125. 
[18] R. Veenhof., "Magboltz - transport of electrons in gas mixtures", version 8.9, http://consult.cern.ch/writeup/magboltz/

[19] A. Bressan et al., "High gain operation of GEM in pure argon", Nucl. Instr. and Meth. A 423 (1997) 119.

[20] A. Bondar et al., "Two-phase argon and xenon avalanche detectors based on Gas Electron Multipliers", Nucl. Instr. and Meth. A 556 (2006) 273.

[21] A. Bondar et al., "Thick GEM versus thin GEM in two-phase argon avalanche detectors", 2008 JINST 3 P07001.

[22] R. De Oliveira, "MPGD technologies", Talk given at the Workshop on Micropattern Gas Detectors (2007), See http://indico.cern.ch/ conferenceOtherViews . py?view=standard\&conf $\mathrm{Id}=16213$.

[23] A. Breskin, R. Alon, M. Cortesi et al., "A Concise review on THGEM detectors," Nucl. Instrum. Meth. A598, 107-111 (2009). arXiv:0807.2026 [physics.ins-det]].

[24] E. Buckley et al., "A Study Of Ionization Electrons Drifting Over Large Distances In Liquid Argon," Nucl. Instrum. Meth. A275, 364-372 (1989). 